\title{
Chapter Head Course in China: Connotation, Value, and Teaching Strategies
}

\author{
Li Bing \\ Qingdao University, Qingdao, China
}

\begin{abstract}
Chapter head course in China is also called “chapter beginning course”, including chapter head chart and chapter introduction. As the prelude of the whole chapter, chapter head course has rich educational value. It is beneficial to provide students with preparatory knowledge and lay the foundation for chapter contents; highlight the teaching direction and optimize the cognitive structure; cultivate students' practical spirit and promote all-round development, etc. The specific implementation strategies of chapter head course teaching are as follows: improve teachers' teaching consciousness and correct the teaching attitude of chapter head course; improve the analytical power of teaching materials and build the teaching framework of chapter head course; combine the teaching art skillfully; enhance the vitality of chapter head course teaching; and so on.
\end{abstract}

Keywords: chapter head course, connotation, value, teaching strategies, core literacy

\section{Introduction}

In China, the Mathematics Curriculum Standard for Full Time Compulsory Education (2011 Edition) has chapter head chart and chapter introduction at the beginning of each chapter as the entry point of this chapter. It reveals the main content and structure framework of this chapter, which not only expands the knowledge learned before, but also provides the preparatory knowledge for the content of this chapter, and plays a guiding role in the learning of this chapter. However, many teachers do not pay attention to this part of the teaching in the actual teaching, ignoring, desalinating, and dealing with the phenomenon of chapter teaching widely exists. Therefore, students seldom take the initiative to learn this part of the content, which undoubtedly causes a waste of teaching resources. Based on this, the author intends to discuss the connotation, value, and implementation strategy of chapter head course teaching, in order to help teachers fully understand the teaching value of chapter head course, arouse teachers' attention to its teaching, and promote teachers to actively and consciously carry out chapter head course teaching in classroom teaching.

\section{The Connotation and Characteristics of Chapter Head Course}

\section{The Connotation of Chapter Head Course}

As the prelude of the whole chapter, chapter head course has a very rich connotation. Chapter head course is also called "chapter beginning course", which is generally composed of chapter head chart and chapter introduction. Most of the pictures at the beginning of the chapter are pictures of real life, which reflect the relationship between the content of this chapter and real life, guide students to connect mathematical

Li Bing, bachelor, College of Mathematics and Statistics, Qingdao University. 
knowledge with real life, and feel the universality of mathematical application, so as to enable students to consciously use mathematical methods to solve problems in real life and deepen their understanding of mathematical application. The introduction of the chapter is an overview of the content of this chapter, outlines the knowledge framework of the whole chapter, expands the humanistic background and mathematical phenomena related to this chapter for students, leads students to feel the universality of the application of mathematics, enables students to initially recognize and understand the content of this chapter, and has an important foreshadowing effect on the subsequent content.

Chapter head course leads the whole chapter, usually does not cover the contents of this chapter in detail, but intuitively and concisely indicates which aspects and mathematical methods will be used in this chapter to study which new mathematical problems.

Taking the third chapter "Linear Equation in one Unknown" in the First Volume of the Seventh Grade of Mathematics in Human Education Edition as an example, the head chart of the chapter is that two cars are driving rapidly on a highway one before and one after the other, creating a mathematical scene of life, and pointing out the application of equation of linear equation in one unknown in real life. Chapter introduction is divided into four paragraphs: The first section reviews the simple equations learned in primary school, helps students to mobilize the original cognitive experience, reviews the form of the equations, and establishes the connection between the new and old knowledge; The second paragraph shows that when studying some problems, people often use equations to solve them, showing the application of the content of this chapter in real life, so that students can understand that learning mathematics is not only the need of learning, but also the need of life, and that mathematics comes from life and serves life; In the third paragraph, two questions are put forward to lead to the content to be explored in this chapter. One is how to turn on a series of equations according to the quantity in the question? How to solve the equation? These two questions point directly to the learning theme of this chapter, provide the preparatory knowledge for students to learn the content of this chapter, point out the learning objectives, point out the learning direction, and help students grasp the key points and difficulties of this chapter; And the last paragraph puts forward the learning expectation for the students. The author hopes that through learning the content of this chapter, we can further feel the value of the equation, infiltrate the mathematical thinking method, learn to use the method of solving problems with the equation of one variable and one order, cultivate the students' awareness of solving practical problems with the mathematical knowledge, and infiltrate the mathematical thinking method. Based on this, teachers can start the teaching design from the chapter head chart, introduce the situation around the relationship between the distance, speed, and time of the two cars, and mathematicize the life problems, so as to lead to the linear equation in one unknown. "One of the important ideas to solve mathematical problems is mathematical modeling, and equations are very important” (Cui, 2019). The cultivation of students' modeling consciousness is in line with the development requirements of students' mathematical modeling and intuitive imagination. According to the implicit teaching objectives and the internal relations of the chapter, the paper depicts the model of quantitative relations in real life, realizes the transition from formula to equation, and enhances the consistency of students' thinking.

\section{Characteristics of Chapter Head Course}

There are many similarities between chapter head course and the review lesson. It not only follows the general rules of review class, but also has its own uniqueness. It is shown in the following three aspects: 
Diversity. "Pluralistic teaching is a kind of teaching thought and a reflection of pluralistic teaching idea in teaching” (Tang, 2006). Pluralistic teaching is an important prerequisite for the cultivation of students' innovative quality, which is conducive to the cultivation of students' core literacy. The diversity of chapter head course is reflected in the following aspects: First, there are multiple forms. Chapter head course consists of chapter introduction and chapter introduction diagram, some of which include tables and knowledge framework, rather than a single combination of chapter introduction diagram and chapter introduction. It is helpful to improve students' imagination ability, inject emotion, and cultivate students' creative consciousness; and second, there are multiple contents. Chapter head course includes not only mathematical knowledge, but also mathematical background, mathematical ideas, and mathematical methods related to mathematical knowledge. The integrated knowledge is an organic whole, which contains history, culture, thought method and humanistic spirit that no single knowledge teaching can match. It is beneficial to deepen students' cognitive understanding from multiple perspectives and effectively expand students' knowledge vision.

Overall planning. "Overall planning refers to unified and comprehensive planning and arrangement. It is an important method to deal with complex problems” (Kuang, 2014). Chapter head course does not exist in isolation. It is the link between the contents of this chapter and the contents previously learned. It is helpful for students to find the connection between the new and old knowledge, and to coordinate the new and old knowledge. This means that chapter head course should not only coordinate the relationship between the topics in this chapter, but also connect the content of this chapter with the content of the previous chapters, so as to help students master the knowledge systematically and comprehensively, review the content of the previous chapters while learning new knowledge, and help students form a complete knowledge vein structure, instead of learning one chapter and forgetting one chapter, resulting in knowledge fault. In addition, the program of this chapter can be extracted from chapter head course. It is systematically introduced that this chapter needs to master mathematical knowledge, mathematical thought, mathematical method, and mathematical emotion, coordinate the connection among various teaching contents of this chapter, and establish a unity of mathematical knowledge, thought, emotion, and method.

Effectiveness. Teacher Cheng Xiaotang, School of Foreign Languages, Beijing Normal University, in the special report "Effectiveness of Primary School English Classroom Teaching”, pointed out that the so-called "effectiveness is whether things have achieved the actual effect in the matter of doing so" (Yan, 2013). Many junior high school students generally have the phenomenon of "As soon as you understand, as soon as you read, as soon as you do it, you will be wrong” in mathematics learning. This phenomenon reflects: The basic knowledge of students is scattered, the logical relationship among knowledge is not clear, and there is no good cognitive structure. The students' thinking ability is weak, and they cannot use the knowledge and skills to solve problems flexibly. Some students cannot sort out the relationship among the sections of knowledge after learning a chapter, even cannot understand what they have learned in this chapter, let alone summarize the content of this chapter, and solve the problem with the knowledge they have learned. However, chapter head course can provide the knowledge framework of this chapter, help students to sort out the logic of knowledge and form a good cognitive structure. Chapter introduction and chapter head chart can reflect the connection between the content of this chapter and the actual life, cultivate the students' spirit of inquiry and the awareness of raising questions about the phenomena in life, improve the students' ability of using mathematical knowledge to solve life problems, and help to cultivate the students' core literacy. 


\section{The Value of Chapter Head Course Teaching}

Chapter head course has rich educational value. It can not only effectively improve students' cognition, but also benefit students' all-round development in many aspects.

\section{Provide Preliminary Knowledge and Prepare Chapter Contents}

"According to Ausubel, a famous American educational psychologist, meaningful learning refers to the process that the new knowledge represented by symbols establishes a non-human and substantive connection with the existing appropriate concepts in the cognitive structure of learners. All meaningful learning is based on the original learning” (Shi, 2016). Chapter head course has the function of connecting the preceding and the following. It can not only carry on the content of the previous study, but also lead to the theme clues and internal relations of this chapter, that is, the "first organizer" of this chapter content, to help students assimilate new knowledge. The picture of life provided by chapter head chart can not only help students to connect the knowledge of this chapter with the reality of life, but also serve as the background map of the introduction of the chapter, beautify the layout of chapter head course, improve students' understanding of beauty and cultivate students' sentiment. For example, the chapter head chart of Chapter 5 "Intersection Line and Parallel Line” in the Second Volume of Mathematics Textbook of Grade 7 in Human Education Edition is a crisscross overpass, which points to the application of intersection line and parallel line in real life, expands the basic plane figure learned in the previous chapter, and consolidates the plane figure learned in the previous chapter. Teachers can take advantage of the students' interest in discussing the overpass to introduce the intersection line and parallel line, from the life situation, with the help of the position and quantity relationship of the angle formed by the intersection of one straight line and the other two straight lines, to study the judgment and nature of the parallel line. Students can learn the method of drawing mathematical conclusions through simple reasoning, cultivate their thinking habits with evidence, conform to their cognitive laws, have a good guiding role in the whole chapter, deepen their impression of intersecting lines and parallel lines, and cultivate their four abilities (the ability to find problems, the ability to put forward problems, the ability to analyze problems, and the ability to solve problems ability).

\section{Highlight Learning Direction and Optimize Cognitive Structure}

"The basic point of view of Bruner's structuralism teaching theory points out that we should attach importance to the development of students' cognitive structure and the knowledge structure of the subject” (Yang, 2008). Chapter head course can help students to make clear their learning objectives grasp the key points and difficulties of this chapter, understand the relationship between the content of this chapter and the knowledge they have learned before, and form a knowledge network, rather than one-sided grasp of the knowledge they have learned. Therefore, it is necessary to teach chapter head course, then learn the content of this chapter, so as to provide psychological preparation for students, conform to the cognitive law of students, optimize the cognitive structure of students, and help students to spiral up and gradually improve on the basis of existing knowledge. Chapter head course also plays a leading role in making the students have a general understanding of the content of this chapter, so as to make the later learning activities more smoothly. For example, the chapter head of Chapter 28 "Acute Triangle" in the second Volume of the Ninth Grade Mathematics Textbook of the People's Education Edition is the Leaning Tower of Pisa in Italy, and the chapter introduction is the introduction of the Leaning Tower of Pisa. The teacher can first expand the relevant stories

of the Leaning Tower of Pisa to attract the students' attention. Then, ask the students whether they can use the 
angle of the center line of the tower and the vertical centerline to describe the degree of inclination of the Leaning Tower of Pisa, highlighting the contents of this chapter, so that the problem can be changed from abstract to concrete, and the students' cognitive structure can be optimized, so that students can think positively and improve the efficiency of classroom learning.

\section{Cultivate Practical Spirit and Promote All-Round Development}

Mathematical core literacy includes mathematical abstraction, logical reasoning, mathematical modeling, intuitive imagination, mathematical operation, and data analysis. Students are required to learn certain knowledge, master sufficient methods and abilities to solve problems and be proficient in using them, in real life, if students encounter problems that need to be solved, students can think about transformation problems from a mathematical perspective. The life pictures provided by the chapter head chart can connect the content of this chapter with the actual life, improve the students' awareness of mathematics application, and help to cultivate the students' practical spirit and core literacy. Chapter introduction reveals "Why we should learn the content of this chapter", "What knowledge we need to master to learn the content of this chapter", "How to learn the content of this chapter", and "How to use it", so as to provide students with preparatory knowledge to learn the content of this chapter and help them form a general understanding of the content of this chapter. For example, the chapter head of Chapter 27 "Similarity" in the Second Volume of the Ninth Grade Mathematics Textbook of Renjiao Edition is the Great Wall. The introduction of chapter gives some examples of figures of the same shape seen in daily life. For example, the pentagram stars of different sizes on the national flag and the photos of the same plate of different sizes are all similar figures. The teaching of "Similar" chapter from these figures can cultivate the students' awareness of solving practical problems by applying mathematics knowledge, make them understand that mathematics comes from life and serves life, and maintain their enthusiasm for mathematics learning. The second paragraph of the introduction of the chapter refers to the "all equal triangle" in the previous study, asking several questions related to it. It not only enables students to connect the content of this chapter with the previous knowledge, trains students' mathematical thinking of analogy, but also leads to the content of this chapter to be studied, guides students to imagine the content of this chapter, arouses students' interest in learning, and improves students' classroom learning efficiency and mathematics application consciousness.

\section{The Implementation Strategy of Chapter Head Course}

In order to realize the application value and promote the wide implementation of chapter head course, teachers can promote the implementation of chapter head course through the following strategies.

\section{Improve Teachers' Teaching Consciousness and Correct the Teaching Attitude of Chapter Head Course}

As Johannes Itten said, "If a teacher only uses the methods he has learned in the teacher training college, and does not teach other things to the students except the outline formulated by the authorities, such a teacher is like a pharmacist who dispenses drugs according to the prescription, and will never become a real doctor" (P. William, R. William, Patrick, \& Peter, 2003). In China, chapter head course is a relatively new type of course which has not been widely implemented. Many teachers will ignore the chapter head chart and chapter introduction in the textbook at present. Therefore, teachers should not neglect the research on chapter head course of teaching materials, but should enhance the teaching consciousness, correct the teaching attitude of chapter head course teaching, patiently explore the law and summarize the experience. Chapter head chart 
connects mathematical knowledge with real life and presents the application of mathematics in life. Teachers can start from chapter head chart, guide students to consciously use mathematical knowledge to solve life problems, help students master mathematical thinking methods, develop mathematical thinking, and cultivate students' ability to find and put forward problems from the perspective of mathematics. Teachers can use teaching skills comprehensively to enable students to master subject knowledge, basic skills and receive ideological education.

\section{Improve the Analytical Power of Teaching Materials and Build the Teaching Framework of Chapter Head Course}

"As the core link of school education, primary and secondary school textbooks embody the national educational thoughts and concepts, are the most basic and important carrier of knowledge in school education, the most important basis for teachers to carry out teaching and organize students' activities, and the main source for students to acquire knowledge” (Li, 2014). Therefore, teachers should improve the analytical power of teaching materials. On the one hand, teachers should improve the analytical power of teaching materials, study the teaching materials in depth, of course, also study the chapter head chart and chapter introduction part, which is also the trust and respect for the content of teaching materials, conscientiously understand the design intent of chapter head chart and chapter introduction, and constantly practice and summarize experience in the teaching process, so as to form a regular understanding and to improve the research ability of teachers' own education and teaching strength and curriculum development and construction capacity, and then realize the value of chapter head course. On the other hand, teachers should carry out teaching plans based on the topic clues given in the chapter head chart and chapter introduction, and provide students with some preparatory knowledge according to why, what, how, and how to learn this chapter as well as how to apply it introduced in chapter introduction, so as to help students form a general understanding of the content of this chapter. Therefore, teachers can prepare lessons according to this point, reasonably carry out teaching design, and improve classroom efficiency. In this way, we can give full play to the role of chapter head course and design a wonderful chapter head course.

\section{Skillfully Combining Teaching Art to Enhance the Vitality of the Teaching of Chapter Head Course}

"The history of mathematics research shows that from ancient times to the Renaissance, teaching was basically regarded as an art, and many Chinese and foreign educators mostly discussed teaching from the perspective of art” (Xiao, 1995). In the teaching of chapter head course, teachers can stimulate students" interest in learning mathematics content, enhance students' desire for knowledge and learning motivation, and help students to have a positive learning orientation by telling the historical anecdotes of mathematics, introducing the actual background and application of mathematics knowledge, creating a situation close to students' reality, and designing a reasonable exploration activity, etc. The key of classroom is teaching efficiency. In the process of teaching, teachers should use the knowledge and experience related to teaching to promote students' learning. The beginning lesson of chapter contains the important and difficult points as well as learning objectives of this chapter. It clearly gives the objectives of students' learning this chapter. Teachers need to interpret these objectives, help students to clarify the context of knowledge, transform them into operable objectives, so as to make the teaching content smooth easy to carry out. The core quality of mathematics is not reflected in students' academic examination results in that year, but in their future adult life and career. Therefore, the teacher guides the students to observe the phenomena in life from the perspective of 
mathematics. When they put forward problems from the perspective of mathematics, the students will try to solve these problems with mathematical methods, improve their mathematical modeling and intuitive imagination ability, which is conducive to the development of mathematical core literacy.

\section{Conclusion}

In a word, teachers should correctly understand the value of the chapter head course, correct the teaching attitude of the chapter head course, prepare lessons carefully, enhance the vitality of the chapter head course teaching, and promote the overall development of students. In addition, teachers should try their best to improve teaching consciousness and teaching material analysis and combine the teaching art skillfully, so as to design a wonderful chapter head course.

\section{References}

Cui, Y. L. (2019). Innovating chapter head classroom infiltration modeling idea-Teaching and reflection on chapter beginning course and concept formation. Course Education Research, 8(10), 160.

Kuang, C. P. (2014). Classroom teaching needs "strategy"-Taking physics in junior middle school as an example. Education Research and Review, 6(7), 45.

Li, S. P. (2014). Study on the textbook system of new China. Hunan: Hunan Normal University.

Shi, Z. Q. (2016). On the way and function of “advance organizer”. Mathematics Bulletin, 55(2), 9-12.

Tang, F. G. (2006). Pluralistic teaching and innovation. Education Exploration, 26(12), 5.

William, P., William, R., Patrick, S., \& Peter, T. (2003). Understanding course. Beijing: Educational Science Publishing House.

Xiao, B. R. (1995). Mathematics teaching art and its philosophical basis. Journal of Mathematics Education, 4(4), 36.

Yan, X. H. (2013). A study on the effectiveness of mathematics review teaching in Senior High School (pp. 2-5). Shandong: Shandong Normal University.

Yang, D. (2008). A new understanding of Bruner's structuralism teaching theory. Modern Education Science, 2(12), 88-90. 